\title{
EGF-stimulated AKT activation is mediated by EGFR recycling via an early endocytic pathway in a gefitinib-resistant human lung cancer cell line
}

\author{
YUKIO NISHIMURA ${ }^{1}$, SOICHI TAKIGUCHI ${ }^{2}$, SHIGERU ITO ${ }^{3}$ and KAZUYUKI ITOH ${ }^{4}$ \\ ${ }^{1}$ Division of Pharmaceutical Cell Biology, Graduate School of Pharmaceutical Sciences, Kyushu University, Fukuoka 812-8582; \\ ${ }^{2}$ Institute for Clinical Research, National Kyushu Cancer Center, Fukuoka 811-1395; ${ }^{3}$ Institute of Biomaterials and \\ Bioengineering, Tokyo Medical and Dental University, Tokyo 101-0062; ${ }^{4}$ Department of Biology, \\ Osaka Medical Center for Cancer and Cardiovascular Diseases, Osaka 537-8511, Japan
}

Received November 13, 2014; Accepted December 23, 2014

DOI: $10.3892 /$ ijo.2015.2871

\begin{abstract}
The receptor tyrosine kinase epidermal growth factor receptor (EGFR) and its ligand epidermal growth factor (EGF) are known to play important roles in malignant tumor cells, and the EGFR signaling pathway is one of the most important targets in various tumors, including non-small cell lung cancer (NSCLC). We reported recently that an aberration in certain steps of EGF-stimulated phosphorylated epidermal growth factor receptor (pEGFR) endocytic trafficking from the early endosomes to the late endosomes occurs in the gefitinib-resistant NSCLC cells, in which large amounts of sorting nexin 1 (SNX1) are colocalized with EGFR in the aggregated early endosomes where the internalized pEGFR is also accumulated of these cells. To further investigate the role of SNX1 in EGF-stimulated pEGFR endocytosis, followed by downstream signaling leading to the activation of phosphatidylinositol 3-kinase (PI3K)-the serine/threonine kinase AKT pathway, we examined the effect of depletion of SNX1 knock-down expression by siRNA and an inhibition of targeting membrane recycling using monensin. Using immunofluorescence, we observed an efficient endocytic transport of pEGFR from early endosomes to late endosomes/lysosomes after
\end{abstract}

Correspondence to: Dr Yukio Nishimura, Division of Pharmaceutical Cell Biology, Graduate School of Pharmaceutical Sciences, Kyushu University, 3-1-1 Maidashi, Higashi-ku, Fukuoka 812-8582, Japan

E-mail: ynkio443@gmail.com

Abbreviations: EGF, epidermal growth factor; pEGFR, phosphorylated epidermal growth factor receptor; pAKT, phosphorylated AKT; HGF, hepatocyte growth factor; pMET, phosphorylated MET; SNX1, sorting nexin 1; NSCLC, non-small cell lung cancer

Key words: epidermal growth factor, epidermal growth factor receptor, serine/threonine kinase AKT, sorting nexin 1, endocytosis, EGFR recycling, MET, endosomes/lysosomes, gefitinib, non-small cell lung cancer
EGF-stimulation in the cells transfected with siRNA-SNX1, whereas the delayed endocytic delivery of pEGFR was evident in the siRNA-control-transfected cells. Furthermore, a large amount of endocytosed pEGFR was accumulated in the presence of monensin in the early endosomes of the SNX1 knock-down cells. In western blot analysis, EGF stimulation of both control and cells transfected with siRNA-SNX1 resulted in rapid phosphorylation of EGFR and enhanced AKT phosphorylation. Monensin-dependent inhibition of AKT phosphorylation was stronger in SNX1 knock-down cells than in controls. In contrast, however, monensin had no effect on AKT phosphorylation triggered by activation of the MET receptor tyrosine kinase. Collectively, we suggest that EGF-stimulated recycling of EGFR to the plasma membrane induces downstream signaling leading to AKT phosphorylation. Suppression of EGFR membrane recycling by SNX1 appears to be critical for the activation of EGFR/PI3K/AKT signaling pathway in human lung cancer cells.

\section{Introduction}

The epidermal growth factor receptor (EGFR) is a member of the ErbB family of tyrosine kinases, and plays an important role in the pathogenesis of different tumors. Therefore, therapies that target EGFR function may prove effective as anticancer treatments $(1,2)$. Upon ligand binding, the EGFR dimerizes, and the intracellular tyrosine kinase region is activated; this causes receptor tyrosine autophosphorylation and transphosphorylation of another receptor monomer $(3,4)$. These events lead to the recruitment and phosphorylation of several intracellular substrates and the subsequent transmission of extracellular signals to the nucleus via an intracellular signaling network (4-6). Specifically, the EGFR pathway involves the downstream mediators RAS/MAPK, phosphatidylinositol 3-kinase (PI3K)/serine/threonine kinase AKT, and STAT signaling pathways; this leads to increased cellular survival, proliferation, invasion, and inhibition of apoptosis $(4,7)$.

After EGFR activation, the epidermal growth factor (EGF)-EGFR complexes are internalized via clathrin-coated 
vesicles and delivered to the early endosomal tubulovesicular compartment commonly referred to as the 'sorting endosomes'. In this compartment, ubiquitinated receptors are recognized by the endosomal sorting complex required for transport (ESCRT) machinery; the ESCRT complexes generate multivesicular bodies (MVBs) by packaging cargo into small vesicles that bud off from the limiting membrane into the lumen of the endosomes (8). This sorting pathway terminates EGF/EGFR signaling by delivering receptors to the lysosomes for degradation, a process known as downregulation (4). Alternatively, EGFR is recycled to the plasma membrane by endosomal recycling pathways.

Selective EGFR-tyrosine kinase inhibitors (EGFR-TKIs) such as gefitinib and erlotinib can block the signal transduction pathways implicated in the proliferation and survival of cancer cells (9-12). These EGFR-TKIs are effective in treating non-small cell lung cancers (NSCLCs) that have activating mutations in the EGFR gene $(13,14)$. Although most EGFR mutant NSCLCs initially respond to EGFR inhibitors, the majority of these tumors eventually develop resistance. Amplification of $M E T$ induces gefitinib resistance by driving ERBB3 (HER3)-dependent activation of PI3K, a pathway thought to be specific to EGFR/ERBB family receptors in lung cancer (15). At present, the mechanisms that contribute to gefitinib resistance in the remaining tumors are unknown.

We reported previously that aberrations in certain steps of EGF/phosphorylated epidermal growth factor receptor (pEGFR) endocytic trafficking from the early endosomes to the late endosomes/lysosomes occurs in gefitinib-resistant $E G F R$ wild-type NSCLC cells, whereas endocytosis of EGFR/pEGFR is normal in gefitinib-sensitive EGFR mutant NSCLC cells $(16,17)$. We observed that large amounts of sorting nexin 1 (SNX1) colocalize with internalized pEGFR in aggregated early endosomes (18).

Furthermore, we demonstrated that silencing of endogenous SNX1 by siRNA stimulates endocytosis and the ligand-induced downregulation of EGFR/pEGFR or $\mathrm{MET} / \mathrm{pMET}$, and concomitantly increases pEGFR and phosphorylated MET (pMET) protein expression in gefitinib-resistant cells $(19,20)$. These data indicate that SNX1 negatively regulates EGF-dependent downregulation of EGFR and its phosphorylation via the early/late endocytic pathway in human lung cancer cells. SNX1 was originally identified as a protein that interacts with EGFR (21) and is localized to early endosomes through its phospholipid-binding motif, which is termed the phox homology (PX) domain (22). Together with the observation that SNX1 enhances degradation of the receptor upon EGF stimulation, these data indicate that SNX1 plays a role in endosome-lysosome trafficking $(21,23)$. However, other reports do not support an intracellular colocalization of SNX1 with EGFR or its direct role in EGFR degradation $(24,25)$; therefore, alternative mechanisms must link SNX1 function with EGFR recycling. However, the specifics of such mechanisms remain to be elucidated.

On the basis of these observations, we designed the current study to investigate the role of SNX1 on EGF-stimulated pEGFR endocytosis. We examined the effect of SNX1 knockdown and treatment with monensin (an inhibitor of membrane recycling), on EGF-stimulated EGFR endocytosis, and on phosphorylation of AKT. In a gefitinib-resistant NSCLC cell line, we observed a rapid phosphorylation of EGFR along with a substantial increase in phosphorylated AKT (pAKT) after EGF stimulation. On the other hand, monensin significantly suppressed AKT phosphorylation in the EGF-treated cells. We therefore suggest that EGF-stimulated recycling of EGFR to the plasma membrane is an important step that activates the PI3K-AKT pathway, and that SNX1 is a negative regulator of EGFR recycling.

\section{Materials and methods}

Materials. Texas Red-labeled human transferrin, and SlowFade antifade reagent were purchased from Molecular Probes (Eugene, OR, USA). Recombinant human EGF and hepatocyte growth factor (HGF) were purchased from PeproTech, Inc. (London, UK). Monensin, bafilomycin A1, cycloheximide (CHX), and DAPI were obtained from Sigma (St. Louis, MO, USA). Other chemicals were of reagent grade and were obtained from commercial sources.

Cell culture. Cell line A549 (National Kyushu Cancer Center, Fukuoka, Japan) was cultured in RPMI supplemented with $10 \%$ fetal bovine serum (FBS). Cells were maintained under standard cell culture conditions at $37^{\circ} \mathrm{C}$ and $5 \%$ $\mathrm{CO}_{2}$ in a humid environment. A549 cells transfected with siRNA-control, -SNX1, or -Rab11a were starved for $12 \mathrm{~h}$ with RPMI-1640 without FBS at $37^{\circ} \mathrm{C}$. The serum-starved cells were preincubated with $10 \mu \mathrm{M}$ monensin for $2 \mathrm{~h}$ or $0.17 \mu \mathrm{M}$ bafilomycin A1 for 30 min and then stimulated with EGF $(100 \mathrm{ng} / \mathrm{ml})$ at $37^{\circ} \mathrm{C}$ for the indicated times, after which further analysis was performed.

Small interfering RNA. siRNAs corresponding to the nucleotide sequences of the SNX1 (5'-AAGAACAAGACCAAGAGC CAC-3') and Rab11a (5'-AATGTCAGACAGACGCGAAA AT-3') were purchased from Dharmacon Research, Inc. (Boulder, CO, USA). Scramble sequence was used as a control. A549 cells were transfected with Lipofectamine 2000 (Life Technologies, Inc., Gaithersburg, MD, USA) in the presence of $40 \mathrm{nM}$ siRNAs according to the manufacturer's instructions. Knock-down efficiency was determined by RT-qPCR, and western blot analysis.

RT-qPCR analysis. A549 cells transfected with siRNAcontrol, -SNX1, or -Rab1la were incubated at $37^{\circ} \mathrm{C}$ for $48 \mathrm{~h}$, and total RNA was extracted from each transfectant using an RNeasy RNA isolation kit (Qiagen, Hilden, Germany) according to the manufacturer's instructions. Transcription into cDNA was done in a $20-\mu 1$ volume using ThermoScript RT-PCR System with random hexamer (Invitrogen Life Technologies, Carlsbad, CA, USA) according to the manufacturer's instructions. All PCR reactions were carried out in a final volume of $25 \mu \mathrm{l}$ and were performed in the ABI PRISM 7000 Sequence Detection System (Applied Biosystems, Inc., Foster City, CA, USA) according to the manufacturer's instructions. Sequence-specific primers were quoted from an official website 'PrimerBank' (http://pga.mgh.harvard. edu/primerbank/) for the indicated genes (Table I). The reaction mixture consisted of SYBR Premix Ex Taq (2X) $12.5 \mu 1$, ROX Reference Dye (50X) (both from Takara Bio, Inc., Shiga, 
Table I. Primers for RT-qPCR for SNX1, Rab11a and $\beta$-actin.

\begin{tabular}{ll}
\hline PCR primer & \multicolumn{1}{c}{ Nucleotide sequence } \\
\hline SNX1 & F: 5'-AGCCCCAGCCAACCTATGA-3' \\
& R: 5'-TCAGGATCAGTTATACCGACTGT-3' \\
Rab11a & F: 5'-CAACAAGAAGCATCCAGGTTGA-3' \\
& R: 5'-GCACCTACAGCTCCACGATAAT-3' \\
\multirow{3}{*}{-actin } & F: 5'-CATGTACGTTGCTATCCAGGC-3' \\
& R: 5'-CTCCTTAATGTCACGCACGAT-3' \\
\hline
\end{tabular}

SNX1, sorting nexin 1.

Japan) $0.5 \mu 1,0.2 \mu \mathrm{M}$ of each specific forward and reverse primer, and $9 \mu 1$ of diluted cDNA (equivalent to $0.03-2.85 \mathrm{ng}$ of total RNA). Amplifications were done under standard conditions $\left(10 \mathrm{sec}\right.$ at $95^{\circ} \mathrm{C}$ followed by 40 cycles of $5 \mathrm{sec}$ at $95^{\circ} \mathrm{C}$ and $31 \mathrm{sec}$ at $\left.60^{\circ} \mathrm{C}\right)$. The number of PCR cycles needed to reach the fluorescence threshold was determined in triplicate for each cDNA, averaged and then normalized to a reference gene $(\beta$-actin). A standard curve was generated with serial 3-fold dilutions of a representative cDNA. For all assays tested, the PCR reaction was linear over the range studied (20-40 cycles of amplification). All RT-PCR reactions gave a single band when analyzed by gel electrophoresis.

Antibodies. Alexa Fluor 488-labeled goat anti-mouse and goat anti-rabbit secondary antibodies were obtained from Molecular Probes. Normal rabbit IgG and normal mouse monoclonal IgG1 were purchased from Imgenex Corp. (San Diego, CA, USA) and Angio-Proteomie (Boston, MA, USA), respectively. Normal goat serum was purchased from Sigma. Mouse monoclonal antibody to SNX1 was purchased from BD Biosciences (San Jose, CA, USA). Anti-EGFR, pEGFR, MET, pMET, AKT, pAKT (Ser473), pAKT (Thr308), p44/42 MAPK (MAPK) and anti-phospho-p44/42 MAPK (p-MAPK) antibodies were obtained from Cell Signaling Technology, Inc. (Beverly, MA, USA), and anti- $\beta$-actin antibody was obtained from Sigma. Anti-cathepsin D was affinity-purified by protein A Sepharose CL-4B (Sigma), followed by immunoaffinity chromatography using antigen-conjugated Sepharose $4 B$ as described previously $(26,27)$.

Immunofluorescence microscopy. Immunofluorescence microscopy was described previously (16-20). A549 cells were grown for 2 days on glass coverslips in 6-well plates in RPMI with $10 \%$ FBS. At $48 \mathrm{~h}$ after the transfection of A549 cells with siRNA-control or -SNX1, cells were starved for $12 \mathrm{~h}$ with RPMI without $\mathrm{FBS}$ at $37^{\circ} \mathrm{C}$ and the cells were incubated in the presence of Texas Red-transferrin in prewarmed medium, and the serum-starved cells were then incubated with EGF $(100 \mathrm{ng} / \mathrm{ml})$ at $37^{\circ} \mathrm{C}$ for $30 \mathrm{~min}$ or $1 \mathrm{~h}$. The cells were fixed with $3.7 \%$ formaldehyde in phosphate-buffered saline (PBS), pH 7.4, permeabilized in PBS containing 0.1\% saponin. After washing with PBS, cells were blocked with PBS-10\% normal goat serum. All subsequent antibody and wash solutions contained $0.1 \%$ saponin. The fixed cells were double-stained for pEGFR with anti-pEGFR monoclonal antibody and for cathepsin D with anti-cathepsin D antibody or for LIMPII with anti-LIMPII antibody followed by washes with PBS containing $0.1 \%$ saponin and incubation for $1 \mathrm{~h}$ with the secondary antibodies at $20 \mu \mathrm{g} / \mathrm{ml}$. Each cell line was stained with DAPI to reveal nuclei. Controls for antibody specificity were non-immune normal mouse IgG1 or non-immune normal rabbit IgG. Late endosomes/lysosomes were stained with anti-LIMPII antibody, since the LIMPII protein is distributed within endocytic organelles and is at its highest concentration in the late endosomes/lysosomes, as observed for other lysosomal glycoproteins, namely, lysosomal-associated membrane protein 1 (LAMP1) and $2(28,29)$. The distribution of the labeled proteins was then analyzed by confocal immunofluorescence microscopy of the fixed cells. Slides were mounted with SlowFade antifade reagent and observed on a Zeiss LSM 510 META confocal laser scanning microscope (Carl Zeiss, Oberkochen, Germany), equipped with krypton/argon laser sources. Colocalization of pEGFR and Texas Red-labeled transferrin, cathepsin D or LIMPII was quantified using ImageJ software and MacSCOPE X software (Mitani Corp., Osaka, Japan).

Western blot analysis. Protein samples were separated by sodium dodecyl sulfate (SDS)-polyacrylamide gel electrophoresis (PAGE) and then transferred to polyvinylidene difluoride membranes (Millipore, Billerica, MA, USA). Following blocking, the membrane was blotted with the appropriate antibody, and subsequently, horseradish peroxidase-conjugated anti-mouse or anti-rabbit IgG (GE Healthcare Bio-Sciences Corp., Tokyo, Japan) was applied. The final signal was revealed by ECL chemiluminescence (Pierce Biotechnology, Inc., Rockford, IL, USA). Digital images were analyzed with NIH Image software to measure the density of each band without a saturated signal. For EGF-stimulated pEGFR degradation, A549 cells transfected with siRNA-control or -SNX1 were starved for $12 \mathrm{~h}$ with RPMI without FBS at $37^{\circ} \mathrm{C}$. The serum-starved cells were then pre-incubated for $30 \mathrm{~min}$ in the presence of CHX $(20 \mu \mathrm{g} / \mathrm{ml})$ before incubation with EGF $(100 \mathrm{ng} / \mathrm{ml})$ at $37^{\circ} \mathrm{C}$ for the indicated times. The cells were then washed with ice-cold PBS and lysed, followed by SDS-PAGE and western blot analysis. Monensin $(10 \mu \mathrm{M})$ or bafilomycin $\mathrm{A} 1(0.17 \mu \mathrm{M})$ was added when the cells were incubated with RPMI.

Statistical analysis. Data are expressed as mean \pm SD unless otherwise noted. Significance $(\mathrm{p}<0.05)$ was determined by using Student's t-test, since all data met all the assumptions for parametric statistical analysis.

\section{Results}

Knockdown of SNX1 stimulates pEGFR transport after EGF stimulation in gefitinib-resistant NSCLC cell line. To examine the role of SNX1 in regulating EGF-stimulated EGFR signaling and endocytosis, we knocked down endogenous of SNX1 in the gefitinib-resistant NSCLC cell line, A549. Fig. 1A (left) shows that endogenous SNX1 mRNA was successfully depleted using specific siRNA in these cells; the level of $S N X I$ transcripts was $14.7 \pm 3.0 \%$ of that observed in control cells $(\mathrm{p}<0.001)$. Consistent with this, western blot analysis (Fig. 3B) 


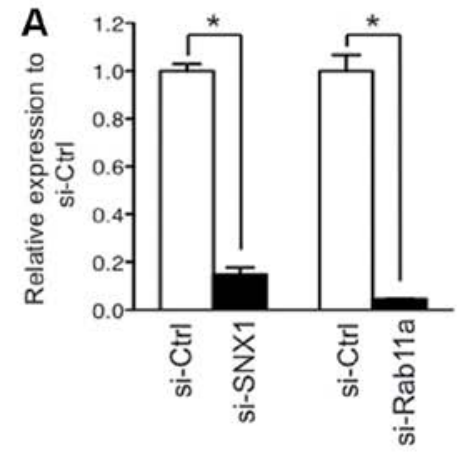

B $30 \mathrm{~min}: \mathrm{pEGFR} / \mathrm{Cat} \mathrm{D}$

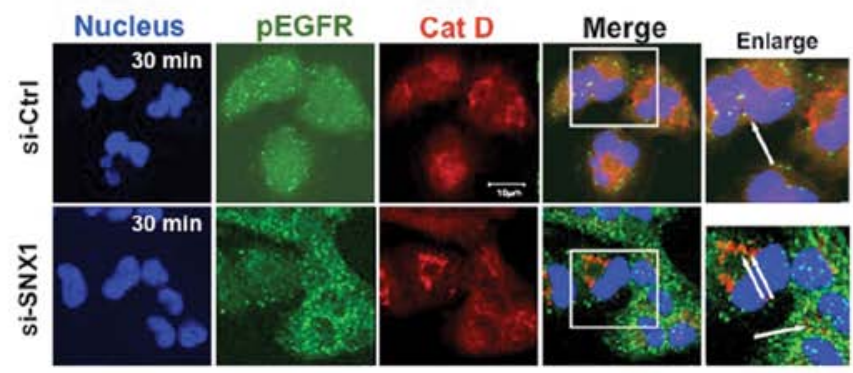

C $60 \mathrm{~min}:$ pEGFR/LIMPII

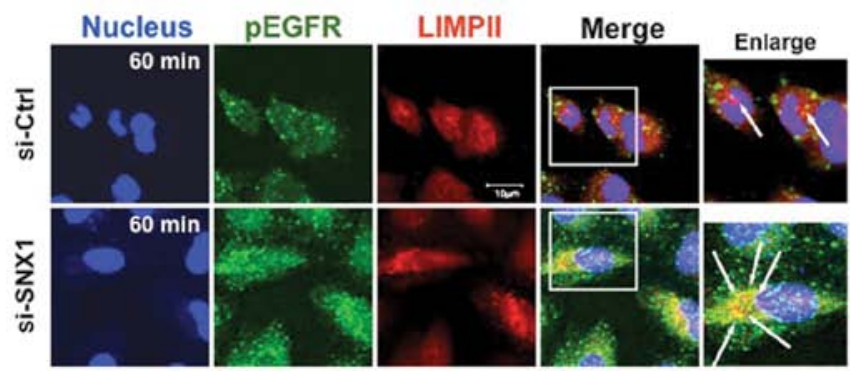

D $60 \mathrm{~min}: \mathrm{pEGFR} / \mathrm{Tf}$

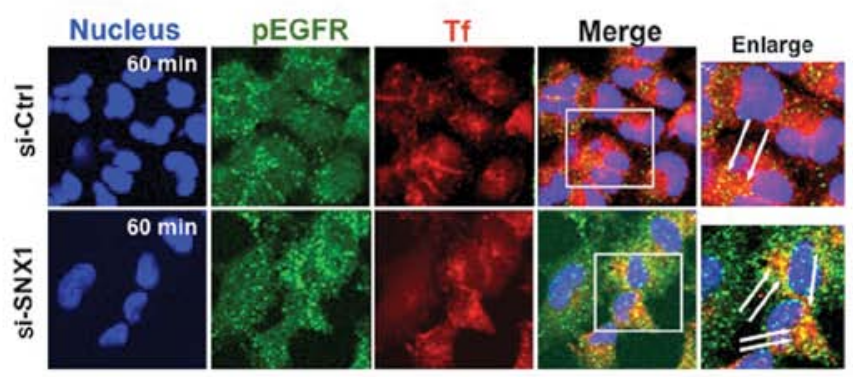

E

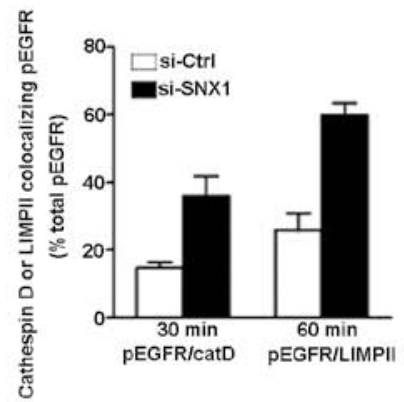

$\mathbf{F}$

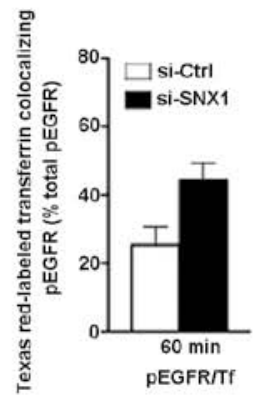

Figure 1. Epidermal growth factor receptor (EGFR) phosphorylation and endocytosis are upregulated in sorting nexin 1 (SNX1)-defient gefitinib-resistant A549 cells. (A) RT-qPCR analysis of SNX1 and Rab11a mRNAs in A549 cells transfected with siRNA-control (si-Ctrl), siRNA-SNX1 (si-SNX1), or siRNA-Rab11a (si-Rab11a). Expression of SNX1 and Rab11a mRNAs was normalized with that of $\beta$-actin. The error bar denotes SD from three separate experiments, and significance ( $(\mathrm{p}<0.05)$ was determined using Student's t-test. (B-F) A549 cells transfected with si-Ctrl or -SNX1 were pre-treated at $37^{\circ} \mathrm{C}$ for $30 \mathrm{~min}$ in the presence of Texas Red-transferrin in prewarmed medium, and the cells were then incubated with epidermal growth factor (EGF) (100 ng/ml) at $37^{\circ} \mathrm{C}$ for 30 or $60 \mathrm{~min}$. Internalized phosphorylated epidermal growth factor receptor (pEGFR), lysosomes, and late endosomes/lysosomes were then detected as described in Materials and methods. Superimposed images of (B) pEGFR (green) with cathepsin D (red) at 30 min, (C) pEGFR (green) with LIMPII (red) at $60 \mathrm{~min}$, or (D) pEGFR (green) with the endocytosed Texas Red-labeled transferrin (red) at 60 min, are also shown. Cells were stained with DAPI (blue) to reveal nuclei. Scale bar, $10 \mu \mathrm{m}$. Right column shows the merged images for double staining of pEGFR (green) and each organelle marker (red), and white squares indicate enlarged regions. The long white arrows indicate the merged confocal images of pEGFR and each organelle marker. (E) Quantitative analysis of colocalization between pEGFR and cathepsin D (at $30 \mathrm{~min}$ ) or pEGFR and LIMPII (at $60 \mathrm{~min}$ ) in the si-Ctrl-transfected cells (white column) or the si-SNX1-transfected cells (black column). Values are the percentage of the integrated density of cathepsin D- or LIMPII-colocalizing pEGFR compared to that of total pEGFR. (F) Colocalization of pEGFR and Texas Red-labeled transferrin in the si-Ctrl-transfected cells (white column) or the si-SNX1-transfected cells (black column) are shown. Values are the percentage of the integrated density of Texas Red-labeled transferrin-colocalized pEGFR compared to that of total pEGFR.

revealed that endogenous SNX1 protein was almost completely depleted following siRNA transfection.

We recently reported that the gefitinib-resistant cells accumulate internalized EGFR in the aggregated early endosomes, whereas the gefitinib-sensitive cells show efficient endocytosis of EGFR, and this is associated with SNX1 (17-19). Accordingly, we inferred that deregulated SNX1 function might underlie the alterations to EGFR endocytosis, leading to gefitinib-resistance in NSCLC cell lines. In order to test this hypothesis, we investigated the fate of internalized pEGFR in early endosomes or late endosomes/lysosomes in A549 cells. Cells transfected with siRNA-control or -SNX1 were incubated with EGF for $30 \mathrm{~min}$ and $1 \mathrm{~h}$, and the colocalization of pEGFR with cathepsin D, LIMPII, or Texas Red-labeled transferrin was then assessed by confocal immunofluorescence microscopy as described in Materials and methods. As shown in Fig. 1B and C, no colocalization of pEGFR with cathepsin D- or LIMPII-positive vesicles was seen in cells transfected with siRNA-control until $1 \mathrm{~h}$ post-EGF treatment. In cells transfected with siRNA-SNX1, however, the amount of pEGFR was markedly induced and a significant increase in the colocalization of pEGFR with cathepsin D and LIMPII 


\section{A Monensin}
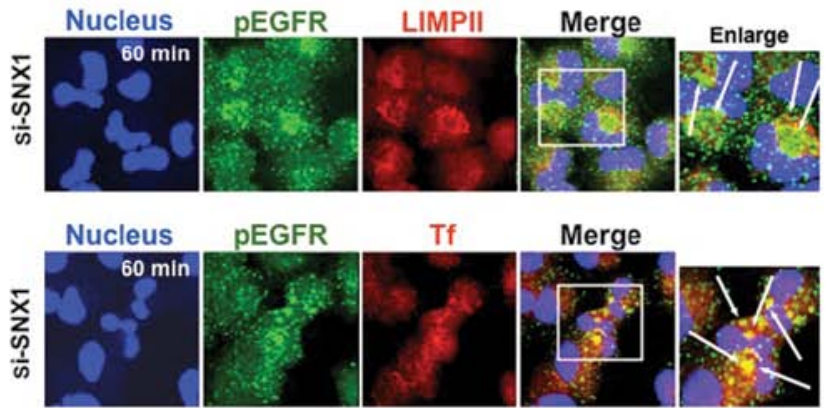

Merge
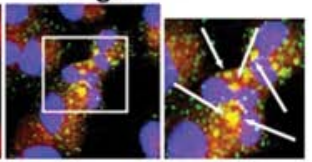

B si-Rab11a
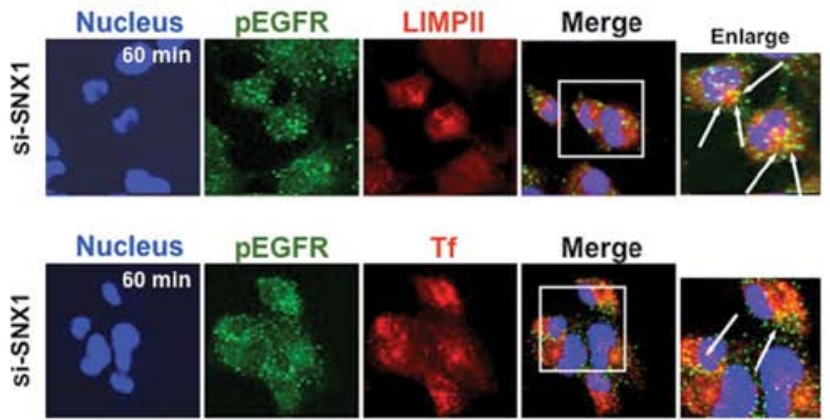

\section{Bafilomycin A1}

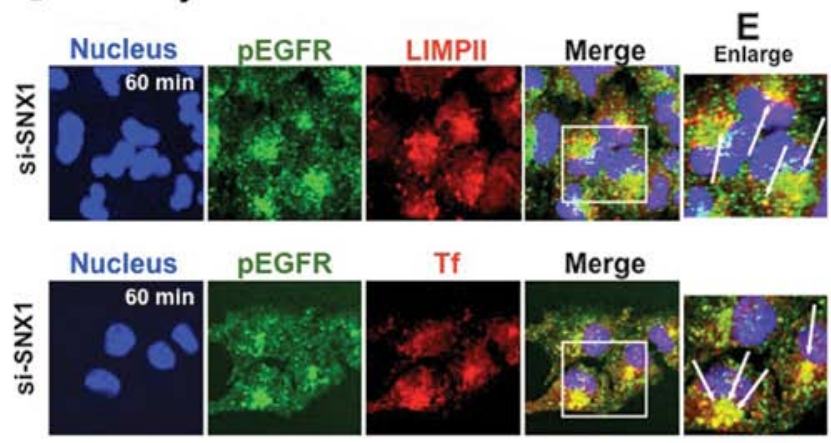

D

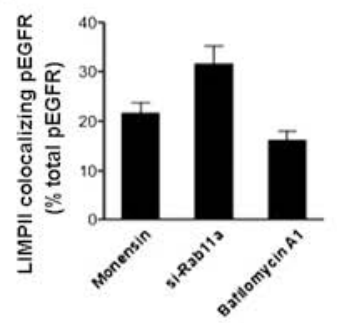

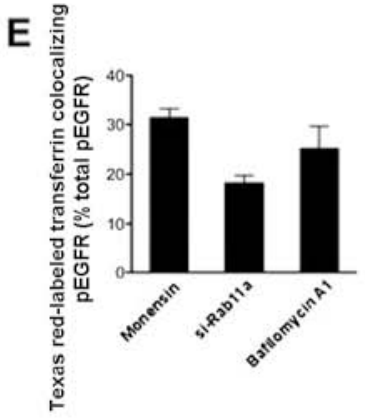

Figure 2. Monensin inhibits epidermal growth factor (EGF)-stimulated endocytosis of phosphorylated epidermal growth factor receptor (pEGFR) in the sorting nexin 1 (SNX1)-deficient cells. SNX1 knock-down cells were pre-treated for (A) $2 \mathrm{~h}$ with monensin, or (C) 30 min with bafilomycin A1. (B) In some cases, cells were transfected with siRNA-targeting Rab1la. Samples were loaded with Texas Red-labeled transferrin (Tf) for 30 min, and then stimulated with EGF $(100 \mathrm{ng} / \mathrm{ml})$ at $37^{\circ} \mathrm{C}$ for $60 \mathrm{~min}$. The cells were double-stained for pEGFR (green) and LIMPII (red) or pEGFR (green) and the endocytosed Texas Red-labeled transferrin (red) as described in Materials and methods. Right column shows the merged images for double staining of pEGFR (green) and each organelle marker (red), and white squares indicate enlarged regions. The long white arrows indicate the merged confocal images of pEGFR with each organelle marker. Cells were stained with DAPI (blue) to reveal nuclei. (D) Quantitative analysis of colocalization of pEGFR and LIMPII in SNX1-deficient cells. (E) The colocalization of pEGFR and Texas Red-labeled transferrin in SNX1-deficient cells. Values are the percentage of the integrated density of LIMPII or Texas Red-labeled transferrin that colocalizes with pEGFR compared to that of total pEGFR. Error bar denotes SD of three separate experiments. It is notable that monensin treatment in the cells caused to increase pEGFR accumulation in the swollen vacuoles that were overlapped with the LIMPII-positive late endosomes and the Texas Red-labeled transferrin-positive early endosomes at $60 \mathrm{~min}$ after EGF stimulation, therefore, indicating that monensin shows a substantial inhibition on the membrane recycling from the early endosomes to plasma membranes and moreover might hamper pEGFR translocation via the endocytic trafficking. It is also shown that bafilomycin A1 treatment blocked the efficient EGF-induced pEGFR endocytosis, leading to an accumulation of pEGFR associated with LIMPII-positive large vacuoles. In contrast, the transfection of siRNA-Rab1la into A549 cells did not lead to an increase of pEGFR and an accumulation of pEGFR-positive large swollen vacuoles after EGF stimulation.

was also observed. Robust accumulation of pEGFR was also observed in the endocytosed transferrin-positive early endosomes of the siRNA-SNX1-transfected cells (Fig. 1D). These results confirm not only that SNX1 depletion increases the amounts of pEGFR, but also that it stimulates ligand-induced pEGFR endocytosis via the early/late endocytic pathway in a gefitinib-resistant NSCLC cell line. These results are consistent with our previous observations (19). Quantitative analysis of the colocalization confirmed that the amount of cathepsin D- or LIMPII-colocalized pEGFR was greater in the cells transfected with siRNA-SNX1 than in control cells after $30 \mathrm{~min}(36.0 \pm 5.8 \%$ vs. $14.8 \pm 1.5 \%$ total pEGFR) and $1 \mathrm{~h}(59.7 \pm 3.6 \%$ vs. $25.8 \pm 4.9 \%$ total pEGFR) of EGF stimulation, respectively (Fig. 1E). In addition, the amount of pEGFR that colocalized with transferrin was greater in cells transfected with siRNA-SNX1 compared to controls after $1 \mathrm{~h}(44.2 \pm 5.1 \%$ vs. $25.4 \pm 5.2 \%$ total pEGFR) of EGF stimulation (Fig. 1F). These data demonstrate that silencing of SNX1 stimulates rapid endocytosis and endocytic trafficking of EGFR/pEGFR via the early/late endocytic pathway in the gefitinib-resistant A549 cells.

Inhibition of membrane recycling leads to accumulation of pEGFR in endosomes in the absence of SNX1. Upon EGF stimulation, a fraction of endocytosed EGFR is recycled back to the plasma membrane instead of being sorted to the late endosomes/lysosomes (30). Therefore, we assumed that non-degraded/non-pEGFR might undergo extensive recycling to the cell membranes from early endosomes via the early endocytic pathway. To further explore the stage at which SNX1 modulates EGFR recycling, we treated SNX1 knock-down cells with monensin, an inhibitor of EGFR recycling between early endosomes and cell membranes (31).

Cell transfected with siRNA-SNX1 were pre-treated with $10 \mu \mathrm{M}$ monensin for $2 \mathrm{~h}$ and followed by stimulation for $1 \mathrm{~h}$ with EGF. Cells were then fixed and double-labeled with antibodies to pEGFR and LIMPII or to pEGFR and the endocytosed Texas Red-labeled transferrin. The change 
A
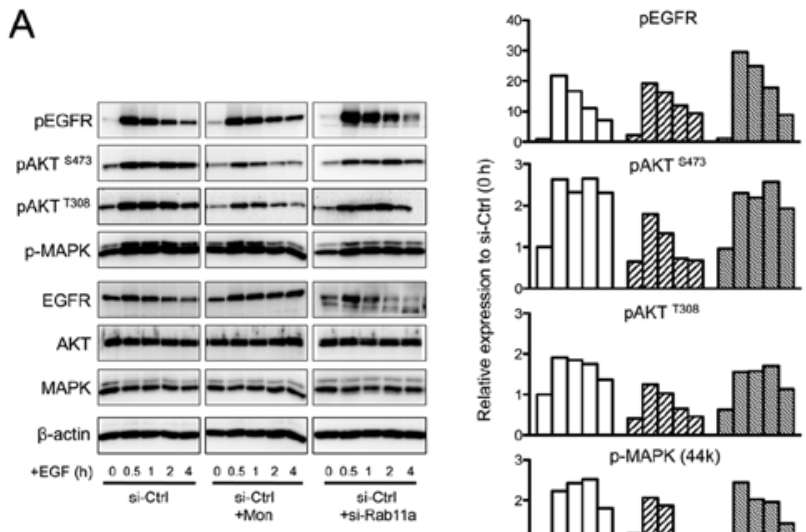

p-MAPK (44k)

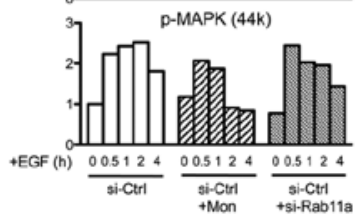

C
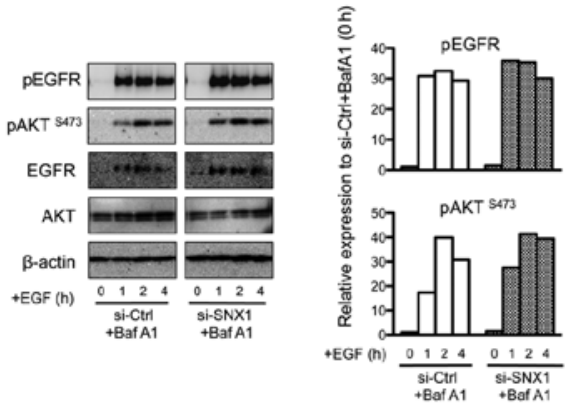

B
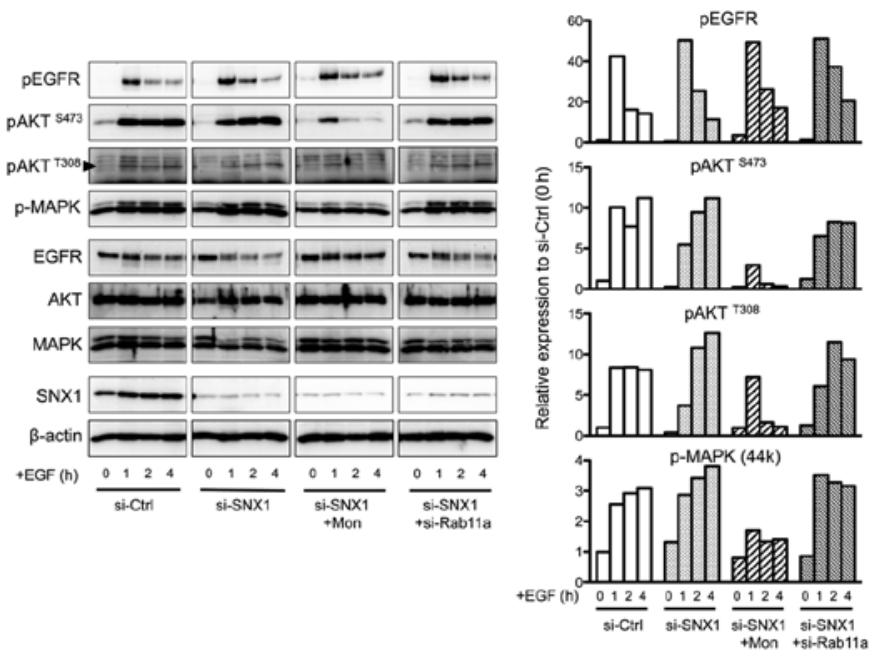

D

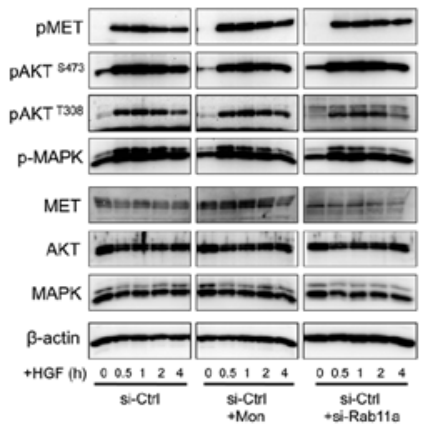

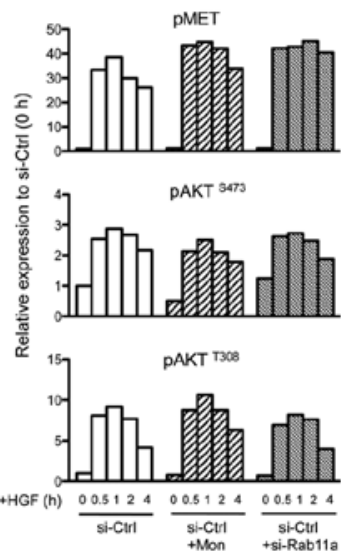

Figure 3. Effect of membrane recycling inhibition on the activation of tyrosine kinase receptors and downstream signaling in sorting nexin 1 (SNX1)-deficient cells. (A) Left: expression of phosphorylated epidermal growth factor receptor (pEGFR), phosphorylated AKT (pAKT) (Ser473), pAKT (Thr308), phospho-p44/42 MAPK (p-MAPK), total epidermal growth factor receptor (EGFR), total AKT, and total p44/42 MAPK (MAPK) before and after 0.5, 1, 2, and $4 \mathrm{~h}$ of epidermal growth factor (EGF) stimulation in the absence or presence of monensin (Mon) $(10 \mu \mathrm{M})$ in A549 cells transfected with siRNA-control (si-Ctrl). Effect of Rab11a knockdown on the activation of growth factor receptors and the downstream molecules in the si-Ctrl-transfected A549 cells was also determined by western blot analysis. $\beta$-actin was used as a loading control. Right: the quantitative western blot analysis is shown. Relative expression levels of pEGFR, pAKT (Ser473), pAKT (Thr308), and p-MAPK are shown and the values are normalized to each phosphorylated protein before EGF stimulation in the si-Ctrl-transfected cells. In the case of p-MAPK, the relative expression levels of phosphorylated form of MAPK (upper band) was determined. All results shown are representative of three independent experiments. pEGFR, phosphorylated human EGFR; pAKT, phosphorylated AKT (protein kinase B). (B) Left: expression of pEGFR, pAKT (Ser473), pAKT (Thr308), p-MAPK, total EGFR, total AKT, and total MAPK before and after 1, 2, and $4 \mathrm{~h}$ of EGF stimulation in the absence or presence of Mon $(10 \mu \mathrm{M})$ in cells transfected with si-Ctrl or -SNX1 was determined by western blot analysis of protein lysates. Effect of Rab11a knockdown on the activation of growth factor receptors and the downstream molecules in the si-SNX1-transfected A549 cells was also determined. pAKT (Thr308) is indicated by arrowhead on the left. Knock-down efficiency of SNX1 was examined by western blot analysis. $\beta$-actin was used as a loading control. Right: the quantitative western blot analysis is shown. Relative expression levels of pEGFR, pAKT (Ser473), pAKT (Thr308), and p-MAPK are shown and the values are normalized to each phosphorylated protein before EGF stimulation in the si-Ctrl-transfected cells. All results shown are representative of three independent experiments. (C) Effect of bafilomycin A1 (Baf A1) on the activation of EGFR and AKT upon EGF stimulation in si-Ctrl-or si-SNX1-transfected cells. Left: the expression of pEGFR, pAKT (Ser473), total EGFR, and total AKT before and after 1,2, and $4 \mathrm{~h}$ of EGF stimulation in the presence of Baf A1 in A549 cells transfected with si-Ctrl or -SNX1. The activation of growth factor receptors and the downstream targets was determined by western blot analysis. $\beta$-actin was used as a loading control. Right: the quantitative western blot analysis is shown. Relative expression levels of pEGFR and pAKT (Ser473) are shown and the values are normalized to each phosphorylated protein before EGF stimulation (in the absence of EGF stimulation) in the si-Ctrl-transfected A549 cells. All results shown are representative of three independent experiments. (D) Left: expression of phosphorylated MET (pMET), pAKT (Ser473), pAKT (Thr308), p-MAPK, total MET, total AKT, and total MAPK before and after 0.5, 1, 2, and $4 \mathrm{~h}$ of hepatocyte growth factor (HGF) stimulation in the absence or presence of monensin in cells transfected with si-Ctrl was determined by western blot analysis of protein lysates. Effect of Rab11a knockdown on the activation of MET and the downstream molecules in the si-Ctrl-transfected A549 cells was also determined by western blot analysis. $\beta$-actin was used as a loading control. Right: quantitative western blot analysis is shown. Relative expression levels of pMET, pAKT (Ser473), and pAKT (Thr308) are shown and the values are normalized to each phosphorylated protein before HGF stimulation in the si-Ctrl-transfected A549 cells. All results shown are representative of three independent experiments.

in the intracellular distribution of pEGFR was examined by confocal immunofluorescence microscopy. Monensin increased pEGFR accumulation in the swollen vacuoles that overlapped with the LIMPII-positive late endosomes after $1 \mathrm{~h}$ of EGF stimulation (Fig. 2A). Robust accumulation of pEGFR was also observed in the endocytosed transferrin-positive early endosomes of the monensin-treated cells (Fig. 2A). These effects were also reproduced by treatment with bafilomycin A1, a lysosomal inhibitor (Fig. 2C). Despite the similarities between monensin and bafilomycin A1, we inferred that pEGFR accumulation induced by monensin was mostly due to the suppression of membrane 
recycling from early endosomes to plasma membranes. We further investigated the role of Rab11a, a small GTPase of the Rab family that regulates the trafficking of intracellular vesicles from the early endosomes through the trans-Golgi network compartments during the recycling process (32). In addition, Rab1la reportedly modulate the recycling of cell surface proteins including the transferrin receptor.

To examine the role of Rab1la on the EGFR recycling in A549 cells, the siRNA-SNX1-transfected cells were depleted of Rab1la using specific siRNA and the intracellular distribution of pEGFR along with early/late endosomes at $1 \mathrm{~h}$ after EGF stimulation was then examined. The depletion of endogenous Rab1la mRNA was verified by RT-qPCR analysis as shown in Fig. 1A; the Rab1la mRNA level was reduced to $4.38 \pm 0.15 \%$ of that observed in controls $(\mathrm{p}<0.001$, Fig. $1 \mathrm{~A}$, right). As shown in Fig, 2B, the stimulation of endocytic delivery of pEGFR from early endosomes to late endosomes triggered by SNX1 knockdown was not affected by the loss of Rab11a. This indicates that Rab11a does not block EGFR recycling, and that pEGFR is mostly recycled back to the plasma membranes from early endosomes, but not from recycling endosomes. Quantitative analysis of the colocalization confirmed that LIMPII/pEGFR colocalization is greater in cells transfected with siRNA-Rab1la than in the cells treated with monensin $(31.4 \pm 3.9 \%$ vs. $21.5 \pm 2.2 \%$ total pEGFR) or bafilomycin A1 (31.4 $\pm 3.9 \%$ vs. $16.1 \pm 1.9 \%$ total pEGFR). Further, the amount of colocalized transferrin and pEGFR was lower in cells transfected with siRNA-Rablla than in the cells treated with monensin $(18.2 \pm 1.6 \%$ vs. $31.3 \pm 1.8 \%$ total pEGFR) or bafilomycin A1 (18.2 $\pm 1.6 \%$ vs. $25.1 \pm 4.6 \%$ total pEGFR) (Fig. 2D and E). These results indicate that endocytosed pEGFR accumulates in early endosomes before being sorted to late endosomes/lysosomes in the siRNA-SNX1-transfected cells treated with monensin or bafilomycin A1. In contrast, pEGFR is sorted to late endosomes/lysosomes efficiently in cells transfected with siRNA-Rab1la.

AKT phosphorylation is inhibited by monensin following EGF stimulation of gefitinib-resistant human NSCLC cells. We described above that the pEGFR was not efficiently trafficked to late endosomes/lysosomes in A549 cells after EGF stimulation, but that SNX1 knockdown increased phosphorylation of EGFR and stimulated its endocytosis via the early endocytic pathway. Since a fraction of internalized non-degraded EGFR is recycled to the plasma membrane from endosomes via the endocytic pathway $(30,31)$, we hypothesized that EGF stimulated EGFR activation and consequent PI3K-AKT pathway might be mediated by recycling of EGFR from early endosomes to the plasma membranes.

To investigate this, we examined whether treatment of SNX1 knock-down cells with monensin affected AKT phosphorylation. Cells transfected with siRNA-control or -SNX1 were pre-incubated with $10 \mu \mathrm{M}$ monensin for $2 \mathrm{~h}$ and then stimulated with $\mathrm{EGF}$ at $37^{\circ} \mathrm{C}$ in the absence or presence of monensin for the indicated times. The lysates were analyzed with anti-pEGFR, anti-pAKT ${ }^{\mathrm{S} 473}$, anti-pAKT ${ }^{\mathrm{T} 308}$, or anti-p-p44/42 MAPK antibody by western blot analysis.

AKT is activated by phospholipid binding, which triggers phosphorylation in the activation loop at Thr308 residue by PDK1, which is essential for AKT catalytic activity (33). On the other hand, PDK2 phosphorylates a Ser473 residue in the carboxy terminus of AKT, which also contributes to activation, which has been identified as mammalian target of rapamycin (mTOR) in a rapamycin-insensitive complex with Rictor and Sin1 $(34,35)$.

As shown in Fig. 3B, the expression of pEGFR in SNX1 knock-down cells was 1.2-fold higher within 1-h incubation than in control cells after EGF stimulation. pEGFR levels then gradually fell during 4-h incubation period (Fig. 3A and B), and the drop was suppressed by bafilomycin A1 (Fig. 3C). This indicates that intracellular degradation of EGF-induced pEGFR proceeds via an endosomal/lysosomal pathway, and is consistent with our previous data (19). These results accordingly imply that SNX1 plays a suppressive role in the phosphorylation and downregulation of pEGFR via the endocytic pathway in the gefitinib-resistant NSCLC cell line A549.

A rapid increase of $\mathrm{pAKT}^{\mathrm{S} 473}$ was seen in the siRNAcontrol-transfected cells, with the level reaching 10-fold that of basal at $1 \mathrm{~h}$; this was sustained during the following 4-h incubation (Fig. 3B). In SNX1 knock-down cells, the increase in $\mathrm{pAKT}^{\mathrm{S} 473}$ was $\sim 5$-fold at $1 \mathrm{~h}$, but reached 10 -fold by $4 \mathrm{~h}$ post-treatment (Fig. 3B). Similarly, the increased levels of $\mathrm{pAKT}^{\mathrm{T} 308}$ were observed in both control and SNX1 knock-down cells, with increments of 8- and 11-fold, respectively (Fig. 3A and B).

Importantly, monensin treatment markedly suppressed AKT phosphorylation; $\mathrm{pAKT}^{\mathrm{S} 473}$ decreased by $\sim 55$ and $85 \%$ after $1 \mathrm{~h}$ of EGF stimulation in the control and SNX1 knock-down cells, respectively (Fig. 3A and B), Similarly, $\mathrm{pAKT}^{\mathrm{T} 308}$ levels were significantly suppressed by monensin, falling to 63 and $85 \%$ of vehicle control following $2 \mathrm{~h}$ of EGF stimulation in control and SNX1 knock-down cells, respectively (Fig. 3A and B). These results indicate that monensin has a stronger inhibitory effect on AKT phosphorylation in the absence of SNX1.

Depletion of Rablla does not inhibit EGF-induced phosphorylation of AKT in gefitinib-resistant human NSCLC cells. We next used siRNA to examine the effect of depletion of Rab11a, which regulates recycling pathway from the recycling compartment to plasma membranes (32), on AKT activation. As shown in Fig. 3A and B, the degree of $\mathrm{pAKT}^{\mathrm{S} 473}$ phosphorylation was unaffected by Rab11a depletion, as it reached 2.5- and 6.5-fold at $1 \mathrm{~h}$ in control and SNX1 knock-down cells, respectively. Also the increased levels of $\mathrm{pAKT}^{\mathrm{S} 473}$ and $\mathrm{pAKT}^{\mathrm{S} 308}$ proteins remained in cell during 4-h incubation. Hence, the Rab11a-dependent recycling pathway is not involved in EGFR-mediated AKT activation. Furthermore, AKT phosphorylation was not affected by treatment with bafilomycin $\mathrm{A} 1$, as $\mathrm{pAKT}{ }^{\mathrm{S} 473}$ reached 39 - and 42 -fold at $1 \mathrm{~h}$ following EGF stimulation in control and SNX1 knock-down cells, respectively (Fig. 3C).

HGF-stimulated AKT phosphorylation is not suppressed by monensin in gefitinib-resistant human NSCLC cells. We next used monensin to determine whether endosomal recycling was involved in signaling from the receptor tyrosine kinase, MET. Cells transfected with siRNA-control were stimulated with $\mathrm{HGF}$ in the absence or presence of monensin at $37^{\circ} \mathrm{C}$ for the indicated times, and then the lysates were analyzed with 
anti-pMET, anti-pAKT ${ }^{\mathrm{S} 473}$, or anti-pAKT ${ }^{\mathrm{T} 308}$ antibody by western blot analysis. As shown in Fig. 3D, MET phosphorylation following HGF stimulation increased 40- and 44-fold in control cells in the absence and presence of monensin, respectively. Furthermore, monensin treatment did not affect phosphorylation of AKT-following HGF treatment, as the increase in pAKT ${ }^{\mathrm{T} 308}$ level was 9- and 12 -fold at $1 \mathrm{~h}$ in the absence and presence of drug, respectively. An increase in $\mathrm{pAKT}^{\mathrm{S} 473}$ was also noted in the monensin-treated cells, and depletion of Rab11a did not inhibit phosphorylation of AKT and MET-following HGF treatment. Together, these data indicate that Rab11a-dependent recycling is not required for EGFR-mediated AKT activation.

\section{Discussion}

Here, we investigated the mechanisms associated with EGFR endosomal recycling, and AKT activation in the gefitinib-resistant NSCLC cell line A549. We verified that knock-down of SNX1 markedly stimulates ligand-induced pEGFR endocytosis via the early/late endocytic pathway, which is accompanied by increasing amounts of pEGFR in cells. This confirms that SNX1 suppresses EGF-stimulated endocytosis of pEGFR via the early/late endocytic pathway. We also found that monensin led to an accumulation of pEGFR in swollen vacuoles that colocalize with LIMPII-positive late endosomes and with the transferrin-positive early endosomes after EGF stimulation. Although these observations are analogous to those of the cells treated with bafilomycin A1, a lysosomal inhibitor, which blocks the efficient EGF-induced degradation of pEGFR, we assumed that pEGFR accumulation in the endosomes of monensin-treated cells might be mostly due to the suppression of membrane recycling from the early endosomes to plasma membranes. Furthermore, knockdown of Rab1la, which regulates intracellular vesicle trafficking from recycling endosomes to plasma membranes, did not affect pEGFR endocytosis or accumulation of pEGFR in the early/late endosomes. Monensin suppresses membrane recycling from early endosomes or recycling endosomes to the plasma membranes (31). Our data accordingly suggest that the majority of endocytosed EGFR/pEGFR in EGF-stimulated cells is recycled back to the plasma membranes from early endosomes, but not from the recycling endosomes.

Furthermore, depletion of SNX1 led to increased expression of pEGFR-following EGF stimulation, and the level of pEGFR then decreased. These observations are consistent with our earlier report (19), and suggest that the early/late endocytic trafficking of EGF-induced pEGFR and subsequent downregulation is accelerated in SNX1 knock-down cells. We conclude that SNX1, either directly or indirectly, is a negative regulator of EGFR/pEGFR endocytosis, and that its downregulation via an ESCRT-dependent pathway is required for activation of the degradation or recycling pathways (8).

We also examined the effect of depletion of SNX1 expression by siRNA or of pharmacological effect such as monensin on the EGF-stimulated AKT phosphorylation by using western blot analysis, and then assessed whether EGF-stimulated AKT phosphorylation might be affected by enhanced recycling. To distinguish the phosphorylation status between the activation loop at Thr308 residue by PDK1 and the carboxy-terminus of AKT at Ser473 residue phosphorylated by the elusive PDK2, two different antibodies, anti-pAKT ${ }^{\mathrm{T} 308}$ and anti-pAKT ${ }^{\mathrm{S} 473}$ antibodies were used for western blot analysis. We found that AKT phosphorylation was rapidly induced in both control and SNX1 knock-down cells after EGF treatment. Importantly, monensin inhibited AKT phosphorylation in both cases, although the effect was much stronger in the absence of SNX1. Thus, SNX1-dependent inhibition of pEGFR recycling from the early endosomes to the plasma membranes is the most probable cause of AKT activation in these cells. Interestingly, depletion of endogenous Rab1la did not alter phosphorylation of either AKT or EGFR. We thus infer that the Rab1la-dependent recycling pathway does not participate in EGF-induced EGFR recycling.

Notably, MET-dependent activation of AKT was not affected by monensin. We infer that MET is not recycled back to the plasma membranes following ligand binding. Rather, we propose that pMET directly activates the PI3K-AKT pathway at the plasma membranes, independently of membrane recycling.

Taken together, our data suggest that upregulation of SNX1 may contribute to the survival of gefitinib-resistant cells by inhibiting endosomal recycling of EGFR to the plasma membranes. This leads to constitutive AKT activation and mTOR signaling; ultimately these signaling cascades positively regulate survival, growth, and proliferation. Thus, targeting the EGFR recycling pathway, in combination with current TKIs, should be considered as a therapeutic strategy, particularly in lung cancer. Further studies are required to delineate the precise molecular mechanisms by which SNX and other proteins regulate the recycling pathways.

\section{References}

1. de Bono JS and Rowinsky EK: The ErbB receptor family: a therapeutic target for cancer. Trends Mol Med 8 (Suppl 4): S19-S26, 2002.

2. Mendelsohn J and Baselga J: The EGF receptor family as targets for cancer therapy. Oncogene 19: 6550-6565, 2000.

3. Carpenter $\mathrm{G}$ and Cohen S: Epidermal growth factor. J Biol Chem 265: 7709-7712, 1990.

4. Yarden Y: The EGFR family and its ligands in human cancer. Signaling mechanisms and therapeutic opportunities. Eur J Cancer 37 (Suppl 4): S3-S8, 2001.

5. Ullrich A and Schlessinger J: Signal transduction by receptors with tyrosine kinase activity. Cell 61: 203-212, 1990.

6. Schlessinger J: Common and distinct elements in cellular signaling via EGF and FGF receptors. Science 306: 1506-1507, 2004.

7. Engelman JA and Cantley LC: The role of the ErbB family members in non-small cell lung cancers sensitive to epidermal growth factor receptor kinase inhibitors. Clin Cancer Res 12: 4372s-4376s, 2006.

8. Henne WM, Buchkovich NJ and Emr SD: The ESCRT pathway. Dev Cell 21: 77-91, 2011.

9. Arteaga CL and Johnson DH: Tyrosine kinase inhibitors-ZD1839 (Iressa). Curr Opin Oncol 13: 491-498, 2001.

10. Barker AJ, Gibson KH, Grundy W, Godfrey AA, Barlow JJ, Healy MP, Woodburn JR, Ashton SE, Curry BJ, Scarlett L, Henthorn L and Richards L: Studies leading to the identification of ZD1839 (IRESSA): an orally active, selective epidermal growth factor receptor tyrosine kinase inhibitor targeted to the treatment of cancer. Bioorg Med Chem Lett 11: 1911-1914, 2001.

11. Baselga $\mathbf{J}$ and Averbuch SD: ZD1839 ('Iressa') as an anticancer agent. Drugs 60 (Suppl 1): S33-S42, 2000.

12. Woodburn JR: The epidermal growth factor receptor and its inhibition in cancer therapy. Pharmacol Ther 82: 241-250, 1999. 
13. Lynch TJ, Bell DW, Sordella R, Gurubhagavatula S, Okimoto RA, Brannigan BW, Harris PL, Haserlat SM, Supko JG, Haluska FG, Louis DN, Christiani DC, Settleman J and Haber DA: Activating mutations in the epidermal growth factor receptor underlying responsiveness of non-small-cell lung cancer to gefitinib. N Engl J Med 350: 2129-2139, 2004.

14. Paez JG, Jänne PA, Lee JC, Tracy S, Greulich H, Gabriel S, Herman P, Kaye FJ, Lindeman N, Boggon TJ, Naoki K, Sasaki H, Fujii Y,Eck MJ, Sellers WR, Johnson BE and Meyerson M: EGFR mutations in lung cancer: correlation with clinical response to gefitinib therapy. Science 304: 1497-1500, 2004.

15. Engelman JA, Zejnullahu K, Mitsudomi T, Song Y, Hyland C, Park JO, Lindeman N, Gale CM, Zhao X, Christensen J, Kosaka T, Holmes AJ, Rogers AM, Cappuzzo F, Mok T, Lee C, Johnson BE, Cantley LC and Jänne PA: MET amplification leads to gefitinib resistance in lung cancer by activating ERBB3 signaling. Science 316: 1039-1043, 2007.

16. Nishimura Y, Bereczky B and Ono M: The EGFR inhibitor gefitinib suppresses ligand-stimulated endocytosis of EGFR via the early/late endocytic pathway in non-small cell lung cancer cell lines. Histochem Cell Biol 127: 541-553, 2007.

17. Nishimura Y, Yoshioka K, Bereczky B and Itoh K: Evidence for efficient phosphorylation of EGFR and rapid endocytosis of phosphorylated EGFR via the early/late endocytic pathway in a gefitinib-sensitive non-small cell lung cancer cell line. Mol Cancer 7: 42, 2008.

18. Nishimura Y, Yoshioka K, Takiguchi S, Bereczky B, Nakabeppu Y and Itoh K: A role for SNX1 in the regulation of EGF-dependent phosphorylated EGFR endocytosis via the early/late endocytic pathway in a gefitinib-sensitive human lung cancer cells. Curr Signal Transduct Ther 6: 383-395, 2011.

19. Nishimura Y, Takiguchi S, Yoshioka K, Nakabeppu Y and Itoh K: Silencing of SNX1 by siRNA stimulates the ligand-induced endocytosis of EGFR and increases EGFR phosphorylation in gefitinib-resistant human lung cancer cell lines. Int J Oncol 41: 1520-1530, 2012

20. Nishimura Y, Takiguchi S, Ito $S$ and Itoh K: Evidence that depletion of the sorting nexin 1 by siRNA promotes HGF-induced MET endocytosis and MET phosphorylation in a gefitinib-resistant human lung cancer cell line. Int J Oncol 44: 412-426, 2014.

21. Kurten RC, Cadena DL and Gill GN: Enhanced degradation of EGF receptors by a sorting nexin, SNX1. Science 272: 1008-1010, 1996.

22. Worby CA and Dixon JE: Sorting out the cellular function of sorting nexins. Nat Rev Mol Cell Biol 3: 919-931, 2002.

23. Zhong Q, Lasar CS, Tronchère $\mathrm{H}$, Sato T, Meerloo T, Yeo M, Songyang Z, Emr SD and Gill GN: Endosomal localization and function of sorting nexin 1. Proc Natl Acad Sci USA 99: 6767-6772, 2002.
24. Carlton J, Bujny M, Peter BJ, Oorschot VM, Rutherford A, Mellor H, Klumperman J, McMahon HT and Cullen PJ: Sorting nexin-1 mediates tubular endosome-to-TGN transport through coincidence sensing of high-curvature membranes and 3-phosphoinositides. Curr Biol 14: 1791-1800, 2004

25. Gullapalli A, Garrett TA, Paing MM, Griffin CT, Yang Y and Trejo J: A role for sorting nexin 2 in epidermal growth factor receptor down-regulation: evidence for distinct functions of sorting nexin 1 and 2 in protein trafficking. Mol Biol Cell 15: 2143-2155, 2004

26. Nishimura Y, Higaki M and Kato K: Identification of a precursor form of cathepsin D in microsomal lumen: characterization of enzymatic activation and proteolytic processing in vitro. Biochem Biophys Res Commun 148: 335-343, 1987.

27. Nishimura Y, Kawabata T and Kato K: Identification of latent procathepsins $\mathrm{B}$ and $\mathrm{L}$ in microsomal lumen: characterization of enzymatic activation and proteolytic processing in vitro. Arch Biochem Biophys 261: 64-71, 1988.

28. Kornfeld S and Mellman I: The biogenesis of lysosomes. Ann Rev Cell Biol 5: 483-525, 1989.

29. Sandoval IV, Arredondo JJ, Alcalde J, Gonzalez-Noriega A, Vandekerckhove J, Jimenez MA and Rico M: The residues Leu(Ile)475-Ile(Leu, Val, Ala)476, contained in the extended carboxyl cytoplasmic tail, are critical for targeting of the resident lysosomal membrane protein LIMP II to lysosomes. J Biol Chem 269: 6622-6631, 1994.

30. Jones MC, Caswell PT and Norman JC: Endocytic recycling pathways: emerging regulators of cell migration. Curr Opin Cell Biol 18: 549-557, 2006.

31. Felder S, Miller K, Moehren G, Ullrich A, Schlessinger J and Hopkins CR: Kinase activity controls the sorting of the epidermal growth factor receptor within the multivesicular body. Cell 61: 623-634, 1990

32. Ullrich O, Reinsch S, Urbé S, Zerial M and Parton RG: Rab11 regulates recycling through the pericentriolar recycling endosome. J Cell Biol 135: 913-924, 1996.

33. Alessi DR, James SR, Downes CP, Holmes AB, Gaffney PR, Reese CB and Cohen P: Characterization of a 3-phosphoinositide-dependent protein kinase which phosphorylates and activates protein kinase $\mathrm{B} \alpha$. Curr Biol 7: 261-269, 1997.

34. Sarbassov DD, Guertin DA, Ali SM and Sabatini DM: Phosphorylation and regulation of Akt/PKB by the rictor-mTOR complex. Science 307: 1098-1101, 2005.

35. Jacinto E, Facchinetti V, Liu D, Soto N, Wei S, Jung SY, Huang Q, Qin J and Su B: SIN1/MIP1 maintains rictor-mTOR complex integrity and regulates Akt phosphorylation and substrate specificity. Cell 127: 125-137, 2006. 